\title{
Determination of phenolic compounds in industrial wastewaters by gas chromatography after extraction and preconcentration by microextraction procedure
}

\author{
Farajzadeh M.A. ${ }^{1,2,}{ }^{*}$, Khiavi E.B. ${ }^{1}$, Feriduni B. ${ }^{1}$ \\ ${ }^{1}$ Department of Analytical Chemistry, Faculty of Chemistry, University of Tabriz, Tabriz, Iran \\ 2Engineering Faculty, Near East University, 99138 Nicosia, North Cyprus, Mersin 10, Turkey \\ Received: 28/08/2018, Accepted: 30/07/2019, Available online: 16/01/2020 \\ *to whom all correspondence should be addressed: e-mail: mafarajzadeh@yahoo.com, mafarajzadeh@tabrizu.ac.ir \\ https://doi.org/10.30955/gnj.002875
}

\begin{abstract}
This paper presents an efficient, simple, and fast method for the derivatization, extraction, and preconcentration of several phenolic compounds (phenol, $o^{-}, m$ - and $p$-cresol, 4-chlorophenol, and 2-nitrophenol) from wastewater samples and analysis of those samples by gas chromatography-flame ionization detection. In this method, initially the phenolic compounds are derivatized with acetic anhydride in an alkaline $\mathrm{pH}$. In the following, the derivatized analytes are extracted into $\mathrm{mL}$-volume of acetonitrile during homogeneous liquid-liquid extraction and further enrichment of the analytes are accomplished by their extraction into $\mu \mathrm{L}$-volume of 1,1,2-trichloroethane through dispersive liquid-liquid microextraction step. Effective parameters controlling the performance of the proposed method such as type and volume of derivatization agent and catalyst, type and volume of extraction/disperser solvent in homogeneous liquid-liquid extraction, and type and volume of extraction solvent and salt addition in dispersive liquid-liquid microextraction are optimized. Under optimum conditions linear range of the proposed method was obtained $0.7-4000 \mu \mathrm{g} \mathrm{L}^{-1}$. Limits of detection and quantification were in the ranges of $0.07-0.20$ and $0.23-0.70 \mu \mathrm{g} \mathrm{L} \mathrm{L}^{-1}$, respectively. Enrichment factors and extraction recoveries were ranged from 220 to 440 and 44 to $88 \%$, respectively.
\end{abstract}

Keywords: Dispersive liquid-liquid microextraction, homogenous liquid-liquid extraction, gas chromatography, derivatization, phenolic compounds, wastewater samples.

\section{Introduction}

Phenols are aromatic components which contain one or more hydroxyl groups that are attached to an aromatic ring. The chemical properties of phenols are unique and are used widely in industry as precursors and components of numerous chemicals in the production of plastics, dyes, drugs, pesticides, antioxidants, paper, and petrochemical products (Nielson et al., 1991). Owing to the increasing production and application of these compounds, they are found in ground waters, rivers, and drinking waters (Visscher et al., 1996). Due to their toxicity, carcinogenicity and persistence, some of them have been included in the lists of priority pollutants of several countries and are required to be determined (Puig and Barcelo, 1996; Commission of the European Communities, 1990). Highperformance liquid chromatography (Ou et al., 2006), electrochemical techniques (Gan et al., 2016; Gan et al., 2019; Gan et al., 2017; Kim et al., 2015), capillary electrophoresis (Fu et al., 2002), and gas chromatography (GC) (Zhou et al., 2005) have been commonly used among other analytical approaches for the trace-level analysis of phenols. However GC with flame ionization detector (FID) (Ghorbanpour et al., 2014; Sarafraz et al., 2012; Farajzadeh et al., 2014), electron capture detector (Bagheri and Saraji, 2001) or mass spectrometry (MS) (Faraji et al., 2009) is preferred to the rest, because of its benefits such as high sensitivity and resolution, fast separation, and low cost (2001; Rodriguez et al., 1997). Extraction of phenolic compounds from an aqueous solution into an organic phase is difficult due to polar nature of them (Pierce, 1968; Halket and Zaikin, 2004). Also, these compounds due to formation of hydrogen bond with the stationary phase of GC column have broad peaks. To resolve the problems of phenolic compounds analysis by GC and to enhance their extractability from an aqueous solution, a derivatization step prior to GC analysis is essential (Ballesteros et al., 1990). For this purpose acetylation in an alkaline aqueous solution by means of acetic anhydride is a simple, cheap and efficient procedure (Rodriguez et al., 1996; Llompart et al., 2002; Sojo and Djauhari, 1999; Turnes et al., 1996). Because of low concentrations of phenolic compounds in the aqueous solutions, sample pretreatment as well as preconcentration of the analytes are crucial steps. Aqueous samples containing phenols were prepared, to separation and preconcentration of the analytes before chromatographic analysis. For this purpose, various methods have been proposed. Liquid-liquid extraction (LLE) (Faraji et al., 2009) and solid phase extraction (SPE) (Zhao et al., 2009) are commonly used as sample 
preparation methods before analysis of phenolic compounds in aqueous samples. These methods are basic sample preparation techniques for a diverse range of samples, but LLE is time-consuming, expensive and hazardous to health due to the high volume of potentially toxic solvents used. Additionally SPE cartridges need pretreatment and still require organic solvents for washing and elution steps. Another extraction method is homogeneous liquid-liquid extraction (HLLE) that extracts the desired solutes existing in a homogeneous aqueous solution into a water-immiscible solvent formed by each kind of phase separation phenomenon (Kujawski et al., 2014). To overcome the limitations of SPE technique, solid phase microextraction (SPME) (2005; Shang et al., 2014), headspace solid-phase microextraction (Bagheri et al., 2008), and stir bar sorptive extraction (Hu et al., 2013) methods were presented as miniaturized SPE techniques. Generally, they are expensive and their fibers (in SPME) are fragile, furthermore, they have a limited lifetime and also the possibility to create sample carryover. To overcome the problems of SPME, liquid phase microextraction (LPME) methods were introduced. Two types of LPME are single-drop LPME (Saraji and Bakhshi, 2005), and hollow fiber LPME (Villar et al., 2012). In 2006, Assadi and coworkers developed a novel LPME technique termed dispersive liquid-liquid microextraction (DLLME) (Rezaee et al., 2006), which consists of a ternary component solvent system. DLLME is a simple and rapid technique with great advantages of low sample volume, low cost, and relatively high enrichment factors.

The aim of this work was to introduce a simple, fast and efficient analytical method for the derivatization, extraction and determination of some phenolic compounds in aqueous samples. In this method, initially the phenolic compounds are derivatized and then extracted by an HLLE method. In the HLLE step, acetonitrile (ACN) is used as an extraction solvent. Organic phase was separated by addition of a salt. The separated layer is used as a dispersant solvent in the following DLLME step and more enrichment is achieved. Effective parameters such as type and volume of derivatization agent and catalyst, reaction time of derivatization, type and volume of extraction/disperser solvent in HLLE, type and volume of extraction solvent in DLLME step, etc will be optimized.

\section{Experimental}

\subsection{Apparatus and chromatographic conditions}

Chromatographic analysis was carried out using a Shimadzu GC-2014 gas chromatograph (Kyoto, Japan) comprising an FID and a splitless/split injector. Separation of the analytes was performed on an HP-5 MS (5\% polydiphenyl, $95 \%$ polydimethyl siloxane) capillary column (30 $\mathrm{m} \times 0.25 \mathrm{~mm}$ i.d., with a $0.25 \mu \mathrm{m}$ film thickness) (Agilent Technologies, CA, USA) Helium (99.999\%, Gulf Cryo, United Arab Emirates) was employed as the carrier gas at a constant linear velocity of $30 \mathrm{~cm} \mathrm{~s}^{-1}$ and make up gas at a flow rate of $30 \mathrm{~mL} \mathrm{~min}$. The injector temperature was constant at $220{ }^{\circ} \mathrm{C}$. Injections $(1 \mu \mathrm{L})$ were done in a splitless/split mode (sampling time of $1 \mathrm{~min}$ and split ratio of $1: 10)$. The oven temperature was regulated as follows: initial temperature $40 \quad{ }^{\circ} \mathrm{C}$ (held for $2 \mathrm{~min}$ ), elevated to $190^{\circ} \mathrm{C}$ at a rate of $10^{\circ} \mathrm{C} \mathrm{min}-1$ and then to more clean up the column enhanced to $220^{\circ} \mathrm{C}$ and held for $5 \mathrm{~min}$. The FID temperature was fixed at $220{ }^{\circ} \mathrm{C}$. A hydrogen generator (OPGU-1500S, Shimadzu, Japan) at a flow rate of $40 \mathrm{~mL} \mathrm{~min}^{-1}$ was used to generate hydrogen gas for FID. The flow rate of air for FID was $300 \mathrm{~mL} \mathrm{~min}^{-1}$. A Hettich centrifuge model D-7200 (Germany) was used for accelerating phase separation. Gas chromatography-mass spectrometry (GC-MS) analysis was carried out on an Agilent $6890 \mathrm{~N}$ gas chromatograph equipped with a 5973 mass-selective detector (Agilent Technologies, CA, USA). The separation was carried out on an HP-5 MS capillary column (30 $\mathrm{m} \times 0.25 \mathrm{~mm}$ i.d., and film thickness of $0.25 \mathrm{~mm}$ ) (Hewlett-Packard, Santa Clara, USA). Helium was used as carrier gas at a flow rate of $1.0 \mathrm{~mL} \mathrm{~min}$. Temperatures of injector and detector and as well as column temperature regulating were the same as used in GC-FID analysis.

\subsection{Reagents and solutions}

All studied analytes (phenol, o-cresol, $m$-cresol, $p$-cresol, 4chlorophenol, and 2-nitrophenol) with a purity of $>98 \%$ were purchased from Merck (Darmstadt, Germany). Deionized water was from Ghazi Company (Tabriz, Iran). Acetic anhydride was obtained from Merck as a derivatization agent. The tested compounds as the catalyst in derivatization reaction (pyridine, picoline, pipyridine, and cyclohexyl amine) were purchased from Merck. Chloroform, 1,2-dibromoethane (1,2-DBE), carbon tetrachloride, and 1,1,2-trichloroethane (1,1,2-TCE) tested as the extraction solvent in DLLME step were from Merck. ACN as the extraction/dispersant solvent was from Merck. Appropriate amounts of aforementioned phenols were dissolved in ACN in order to preparation of a mixture standard solution of the phenolic compounds at a concentration of $1000 \mathrm{mg} \mathrm{L}^{-1}$ (each analyte). Diluted solutions were prepared daily from the standard solution by adding deionized water. A mixture standard solution of the derivatized analytes was prepared by adding $20 \mu \mathrm{L}$ picoline and $100 \mu \mathrm{L}$ acetic anhydride into $1 \mathrm{~mL}$ 1,1,2-TCE containing $1000 \mathrm{mg} \mathrm{L}^{-1}$ of each phenolic compound. This solution was injected into the separation system each day (three times) for quality control, and the obtained peak areas were used in the calculation of enrichment factors (EFs) and extraction recoveries (ERs).

\subsection{Samples}

In order to assess the ability of the proposed method in analysis of the compounds of interest in aqueous samples, the method was applied for determination of the selected phenolic compounds in some wastewater samples. For this purpose, wastewater samples were collected from treatment plants of petrochemical and refinery units. Input and final output of petrochemical unit were obtained from Tabriz Petrochemical Company (Tabriz, Iran). Output of desalination unit and final output of refinery were collected from Tabriz Refinery. The input of the petrochemical wastewater was prepared at a dilution ratio of 1:1 from 
deionized water before applying the proposed method. Other samples were used without any dilution.

\subsection{Derivatization and extraction procedure}

Five $\mathrm{mL}$ aqueous sample or deionized water spiked with $1 \mathrm{mg} \mathrm{L}^{-1}$ of each analyte or sample solution was placed in a $12-\mathrm{mL}$ glass test tube. Forty $\mu \mathrm{L}$ picoline was added as a catalyst for derivatization reaction. For derivatizing of the selected phenolic compounds, $50 \mu \mathrm{L}$ acetic anhydride was added and the resulting mixture was agitated with hand for $30 \mathrm{~s}$. In the following, $2 \mathrm{~mL} \mathrm{ACN}$ containing $25 \mu \mathrm{L}$ 1,1,2-TCE was added and a uniform solution resulted. Then to initiate a two-phase separation, $1.5 \mathrm{~g} \mathrm{NaCl}$ was dissolved into it and centrifuged for $1 \mathrm{~min}$ at $7000 \mathrm{rpm}$. In this step $1.0 \pm 0.05 \mathrm{~mL}$ organic phase was collected at top of the tube. In the DLLME step to increase analytes enrichment, the collected organic phase in the first step was removed by a 2-mL glass syringe and rapidly injected into $5 \mathrm{~mL}$ deionized water containing $0.4 \mathrm{~g} \mathrm{NaCl}$ placed into a $10-\mathrm{mL}$ test tube with conical bottom. A cloudy solution was formed, that resulted from dispersion of the tiny droplets of 1,1,2-TCE into the aqueous solution due to dissolving $A C N$ in water and the derivatized analytes were extracted and concentrated into 1,1,2-TCE. Then, the resultant solution was centrifuged for $5 \mathrm{~min}$ at $7000 \mathrm{rpm}$, which led to sedimentation of the dispersed droplets of the extractant at the bottom of the tube. In the centrifugation step $10 \pm 0.5 \mu \mathrm{L}$ of the organic phase was sedimented. $1 \mu \mathrm{L}$ of the organic phase was withdrawn and injected into the separation system for analysis.

\subsection{Calculation of EFs and ERs}

Two main parameters, namely EF and ER, have been employed for evaluating the proposed method. The EF is defined as the ratio of the analyte concentration in the sedimented phase $\left(C_{\text {sed }}\right)$ to the initial concentration of the analyte $\left(C_{0}\right)$ in the sample:

$$
\mathrm{EF}=C_{\mathrm{sed}} / C_{0}
$$

$C_{\text {sed }}$ is calculated by comparison of the peak areas obtained by direct injection of the standard solution of the derivatized analytes with those obtained by injection of the extractant after performing the proposed method. The ER is defined as the percentage of the total analyte amount $\left(n_{0}\right)$ which is extracted into the sedimented phase $\left(n_{s e d}\right)$ :

$$
\mathrm{ER}=n_{\text {sed }} / n_{0} \times 100=\left(C_{\text {sed }} \times V_{\text {sed }}\right) /\left(C_{0} \times V_{\text {aq }}\right) \times 100=\mathrm{EF} \times V_{\text {sed }} / V_{\text {aq }} \times 100
$$

Where $\mathrm{V}_{\text {sed }}$ and $\mathrm{V}_{\mathrm{aq}}$ are volumes of the sedimented phase in DLLME step and aqueous solution, respectively.

\section{Results and discussion}

In this method, initially the selected phenolic compounds are derivatized by acetic anhydride. In the following, the derivatized analytes are extracted and preconcentrated by coupling HLLE and DLLME procedures. Indeed, the HLLE method provides extraction of the analytes from the aqueous sample and the DLLME stage results in enrichment of the analytes by transferring them into $\mu \mathrm{L}-$ volume of an extraction solvent. To obtain optimum conditions, more effective parameters on the derivatization and extraction efficiencies are investigated.

\subsection{Optimization of derivatization step}

\subsubsection{Type and volume of catalyst}

Derivatization of phenolic compounds by acetylation is usually carried out in an alkaline medium. In this procedure, a basic agent acts as a catalyst. For this purpose, four basic agents including picoline, pyridine, pipyridine, and cyclohexyl amine were tested. The obtained results showed that the selected analytes were not derivatized in the presence of pipyridine. As it can be seen from Figure 1, picoline gives the highest efficiency among the other basic catalyst used. Therefore picoline was selected as the catalyst for the further experiments. In the following, to achieve the optimized volume of picoline, varied volumes of it within the range of $6-60 \mu \mathrm{L}$ were tested. Considering the obtained results, analytical signals were higher in the case of $40 \mu \mathrm{L}$ picoline compared to other volumes. Therefore $40 \mu \mathrm{L}$ was selected as the optimum volume of picoline in the subsequent stages of the optimization process. It seems that volumes less than $40 \mu \mathrm{L}$ were not enough and in the cases of volumes higher than $40 \mu \mathrm{L}$, the sedimented phase volume increased which led to reduced analytical signals due to diluting effect.

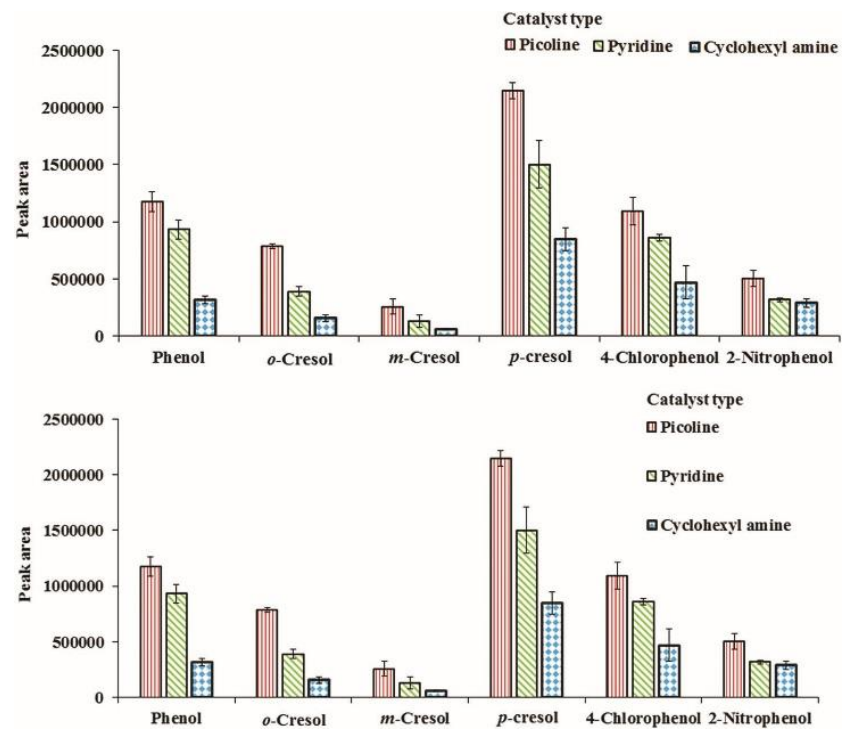

Figure 1. Study of catalyst type on the derivatization efficiency. Extraction conditions: aqueous phase, $5 \mathrm{~mL}$ deionized water spiked with $1 \mathrm{mg} \mathrm{L}^{-1}$ of each phenolic compounds; catalyst volume, $20 \mu \mathrm{L}$; acetic anhydride volume, $100 \mu \mathrm{L}$; acetonitrile volume, $2 \mathrm{~mL}$; extraction solvent, chloroform $(70 \mu \mathrm{L})$; concentration of $\mathrm{NaCl}, 30 \%, w / v$; centrifuging rate, $7000 \mathrm{rpm}$; centrifuging time, $1 \mathrm{~min}$; volume of the collected phase used in

DLLME step, $1.0 \mathrm{~mL}$; aqueous phase in DLLME step, $5.0 \mathrm{~mL}$ deionized water; centrifuging rate in DLLME step, $7000 \mathrm{rpm}$; and centrifuging time in DLLME step, $5 \mathrm{~min}$. The error bars indicate the maximum and minimum of three repeated determinations

\subsubsection{Derivatization reaction time}


To select the optimal derivatization reaction time, different times $(0,0.5,1.0,2.0,4.0,5.0,7.0$ and $10 \mathrm{~min})$ were tested. In this study, the reaction time is defined as the interval spent after mixing the aqueous solution containing the selected analytes with the derivatization reagent (acetic anhydride) and just before adding of the extraction/disperser solvent (ACN). The obtained results in Figure 2 show that the reaction time has no significant effect on the analytical signals. Indeed derivatization of the analytes is very fast. Therefore, the subsequent experiments were carried out without applying excess time for derivatization step.

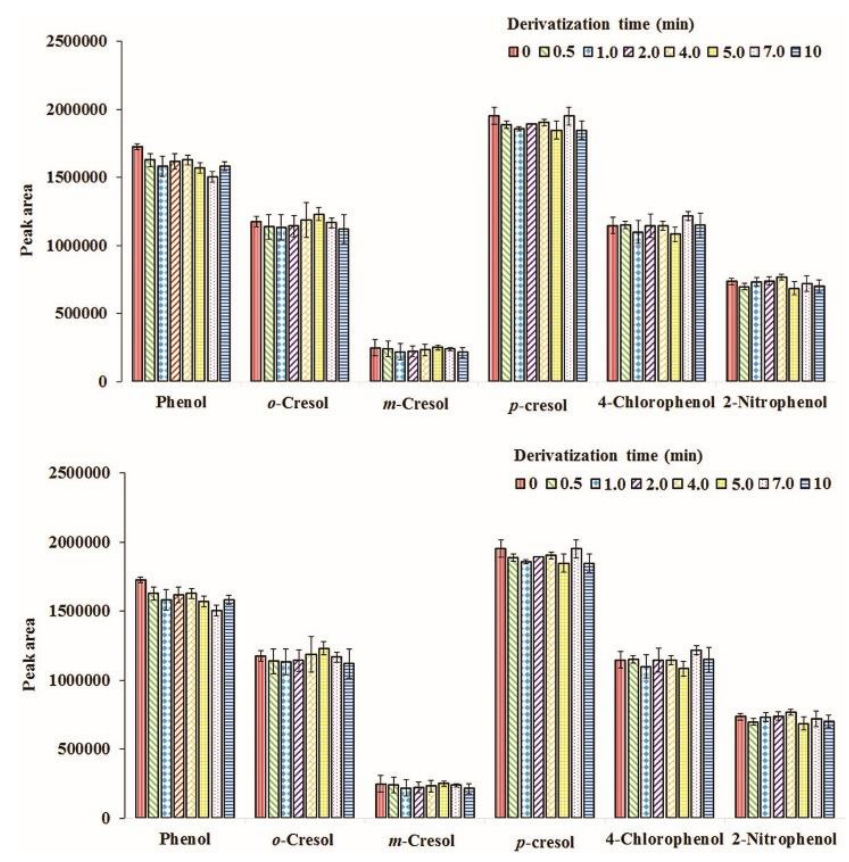

Figure 2. Study of derivatization reaction time. Extraction conditions: catalyst, picoline $(40 \mu \mathrm{L})$; other conditions are the same as used in Figure 1. The error bars indicate the maximum and minimum of three repeated determinations

\subsubsection{Derivatization reagent volume}

To evaluate the effect of acetic anhydride volume on the derivatization efficiency, different volumes of the reagent $(0,5,10,20,50,70,80,100$, and $120 \mu \mathrm{L})$ were tested. The obtained results (Figure 3 ) show that the peak areas increase up to $50 \mu \mathrm{L}$, and then remain constant till $80 \mu \mathrm{L}$ and partially decrease at high volumes. It can be concluded that an inadequate derivatization of the analytes is obtained at low volumes $(<50 \mu \mathrm{L})$ of acetic anhydride. On the other hand, at high volumes $(>80 \mu \mathrm{L})$ of the derivatization agent, the volume of the sedimented phase increased which led to dilution of the analytes. It seems that in this case a portion of acetic anhydride is dissolved in ACN in HLLE step and transferred to DLLME procedure. Therefore $50 \mu \mathrm{L}$ was selected as the optimum volume of acetic anhydride in the subsequent stages of the optimization process.

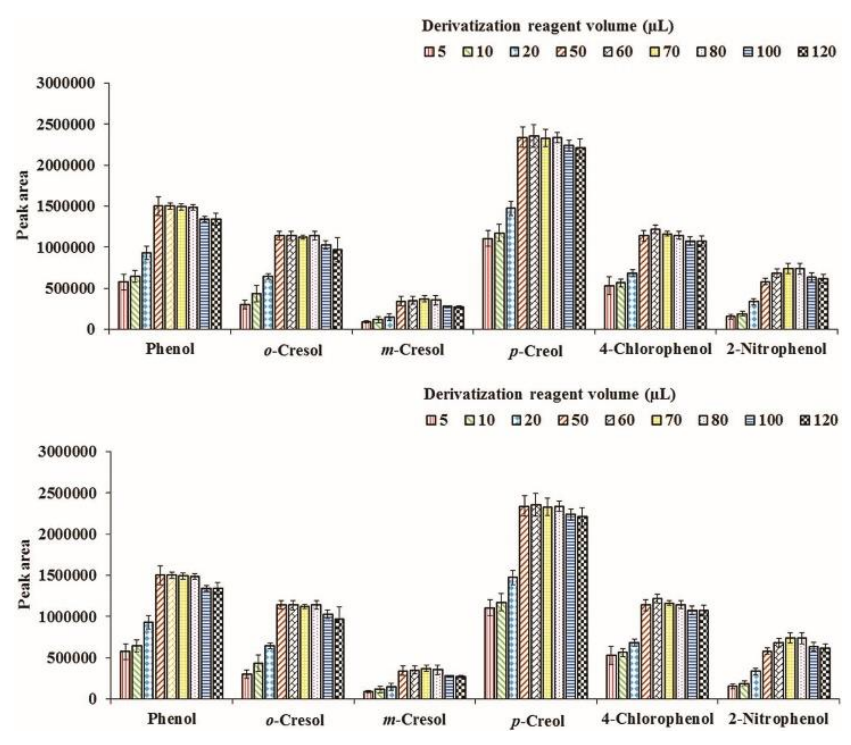

Figure 3. Influence of derivatization reagent volume. Extraction conditions: the same as used in Figure 2 without applying extraction time for derivatization step. The error bars indicate the maximum and minimum of three repeated determinations

\subsection{Optimization of extraction procedures}

\subsubsection{Type and volume of disperser/extraction solvent in the HLLE stage}

Selection of a suitable extraction solvent for the extraction of the derivatized phenolic compounds from the aqueous solution is an important parameter in this method. In this work, the extraction solvent used in HLLE step acts as a disperser solvent in the next DLLME step. This solvent is selected on the basis of its miscibility with the organic phase (extraction solvent of DLLME) and aqueous phase (to form a homogenous solution), its ability to produce a towphase system upon adding a salt, and its high extraction efficiency for the compounds of interest from the aqueous solution. Among the tested solvents (methanol, acetone, $A C N$, and THF), ACN was selected by providing the abovementioned factors. To study effect of ACN volume on the extraction efficiency, different volumes $(0.5,1.0,1.5,2.0,2.5$, and $3.0 \mathrm{~mL})$ were tested. The volumes of the separated phase in the cases of 1.0, 1.5, 2.0, 2.5, and $3.0 \mathrm{~mL}$ were $0.2,0.6,1.0,1.6$, and 2.0 $\mathrm{mL}$, respectively. Also no separated phase was obtained when $0.5 \mathrm{~mL} \mathrm{ACN}$ was used. It is noted that in the 0.2 and $0.6 \mathrm{~mL}$, the collected phase volume was reached to $1 \mathrm{~mL}$ with pure ACN and then applied in DLLME procedure. Also, in the cases of 1.6 and $2.0 \mathrm{~mL}$ collected phase, only $1 \mathrm{~mL}$ of them was utilized for the following DLLME step. Based on the achieved results (Figure 4), ERs increase till $2 \mathrm{~mL}$, and then decrease to corresponding amounts at higher volumes of ACN. Therefore $2 \mathrm{~mL}$ was selected as the optimum volume of $A C N$. 


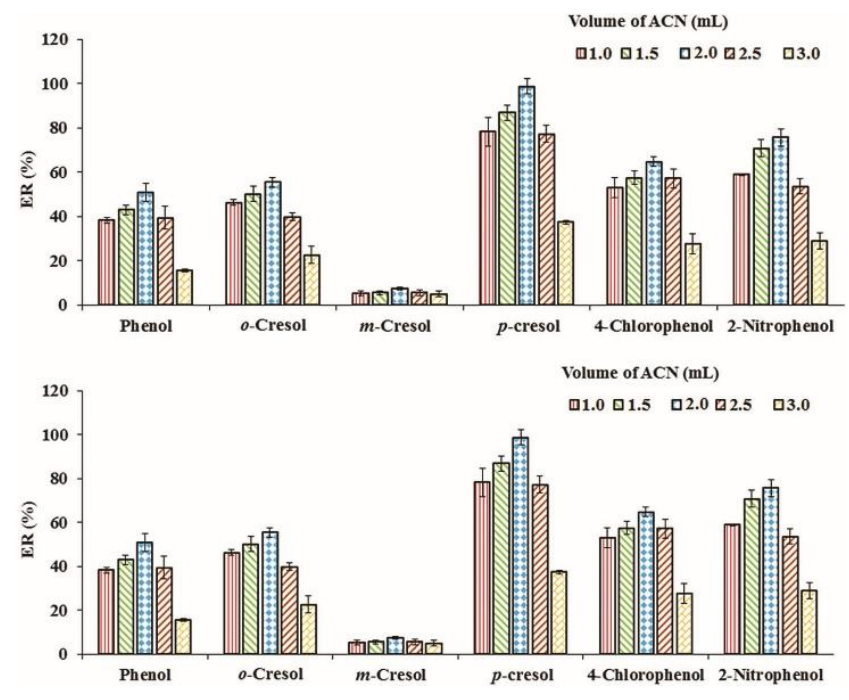

Figure 4. Study of ACN volume. Extraction conditions: the same as used in Figure 3, except $50 \mu \mathrm{L}$ acetic anhydride was used as derivatization agent. The error bars indicate the maximum and minimum of three repeated determinations

\subsubsection{Selection of extraction solvent kind and volume in DLLME stage}

One of the important and effective parameters on the extraction efficiency of the proposed method is the extraction solvent kind in DLLME. The extraction solvent should have the following features: immiscible in water, high ability to extract the analytes, good chromatographic behavior, and preferably higher density than water. For this purpose four organic solvents including chloroform, 1,2-DBE, 1,1.2-TCE, and carbon tetrachloride were tested. To obtain a same sedimented phase volume (50 $\pm 2 \mu \mathrm{L}$ ), $100 \mu \mathrm{L}$ chloroform, $82 \mu \mathrm{L}$ 1,2-DBE, $93 \mu \mathrm{L}$ carbon tetrachloride, and $70 \mu \mathrm{L} 1,1.2$-TCE were used. As it is shown in Figure 5, 1,1,2-TCE is a proper extraction solvent for this stage since it provides high analytical response among the other tested solvents. Therefore, it was selected as the extraction solvent.

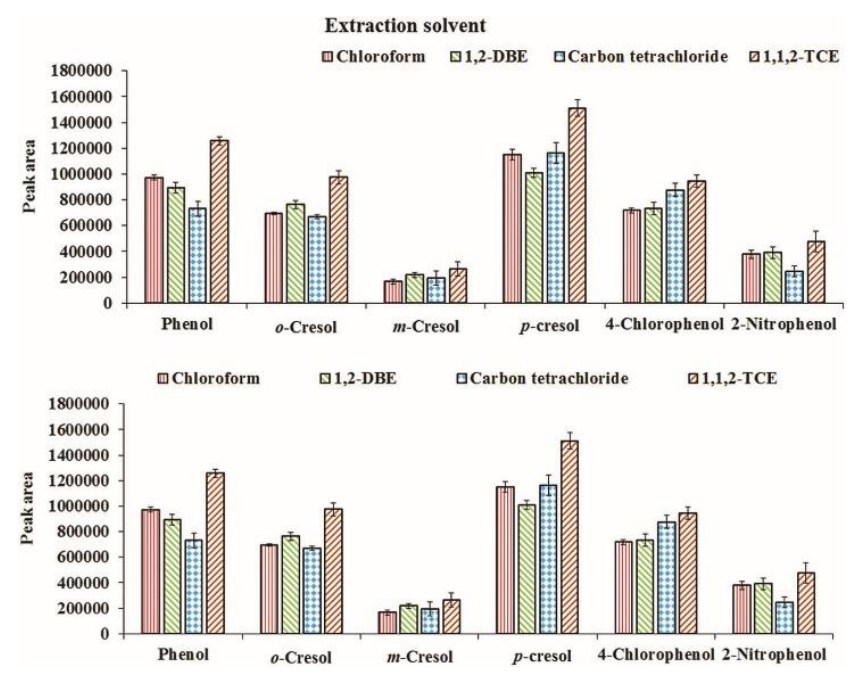

Figure 5. Selection of extraction solvent in DLLME step. Extraction conditions: the same as used in Figure 4, except $2 \mathrm{~mL}$ ACN was used in HLLE step. The error bars indicate the maximum and minimum of three repeated determinations
Volume of the extraction solvent can affect repeatability of the results and EF by changing volume of the sedimented phase. To study the effect of this parameter \different volumes of 1,1,2-TCE $(30,40,50,60,70$, and $80 \mu \mathrm{L})$ were examined. The obtained results showed that by increasing volume of the extraction solvent, the analytical signals decreased. It is noted that by increasing the volume of 1,1,2-TCE from 30 to $80 \mu \mathrm{L}$, the volume of the sedimented phase increased from 10 to $60 \mu \mathrm{L}$. Therefore, $30 \mu \mathrm{L}$ was selected as the suitable volume of the extraction solvent in order to obtain high EFs.

\subsubsection{Investigation of ionic strength effect in DLLME}

Generally, salt addition can have multiple effects on the extraction efficiency which have been addressed as follows: (1) Solubility of the analytes in aqueous phase decreased and their extraction into organic phase enhanced which improves extraction efficiency, (2) solubility of the extraction solvent in aqueous phase was decreased which leads to increase in volume of the sedimented organic phase, and (3) viscosity of the aqueous phase was increased which leads to decrease in diffusion coefficients of the analytes and low ERs are obtained. To investigate the effect of salt addition, varied values of sodium chloride within the range of $0,4,8$, and $12 \%, w / v$ were investigated. To access a same volume of the precipitated phase $(10 \pm 0.5 \mu \mathrm{L}), 30,27,25$, and $20 \mu \mathrm{L}$ of the extraction solvent (1,1,2-TCE) were used for $0,4,8$, and $12 \%, w / v$, of salt, respectively. The results (Figure 6) indicate that the extraction efficiency increases up to $8 \%$, $w / v$, and then decreases at high concentrations of the salt. Therefore $8 \%, w / v, \mathrm{NaCl}$ was selected for the further studies.
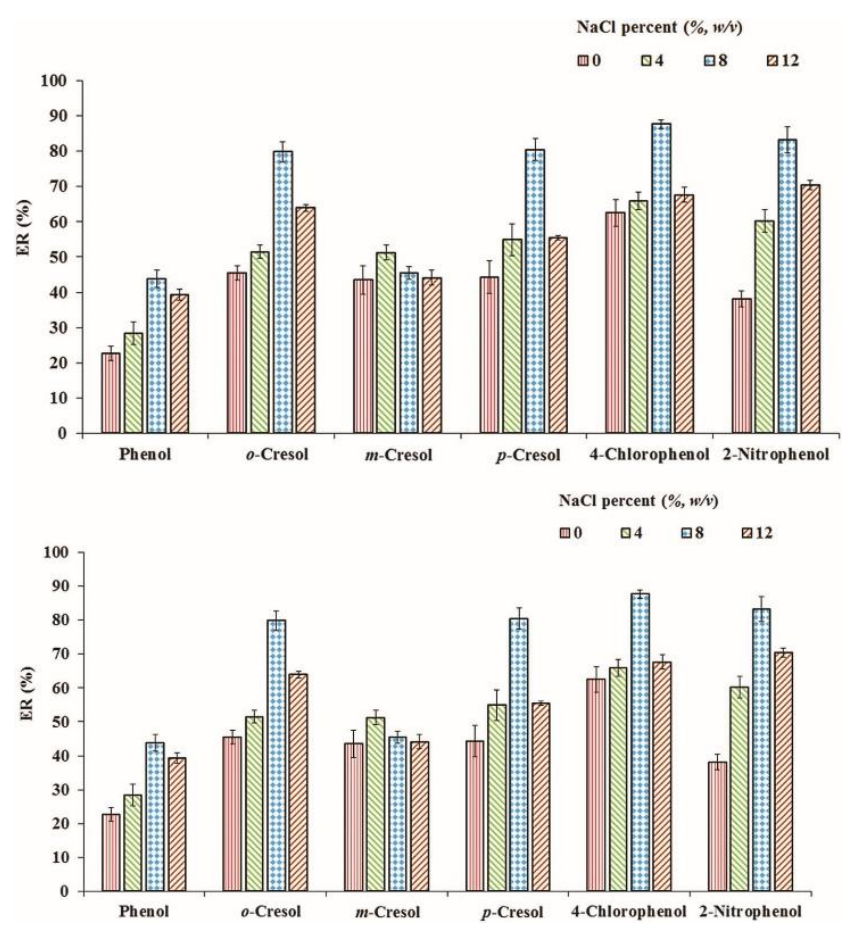

Figure 6. Study of ionic strength in DLLME. Extraction conditions: the same as used in Figure 5, except $30 \mu \mathrm{L}$ 1,1,2-TCE was used. The error bars indicate the maximum and minimum of three repeated determinations 


\subsubsection{Evaluation of analytical performance of the method}

The performance of the proposed method in analysis of the selected phenolic compounds was assayed under the obtained optimum conditions by calculation of linear range (LR), coefficient of determination, limit of detection (LOD), limit of quantification (LOQ), precision expressed as relative standard deviation (RSD\%), EF, and ER. These results are summarized in Table 1 . According to the results, the RSD values are equal or less than $8 \%$ for intraand inter-day precisions which indicate that an acceptable Table 1. Quantitative features of the proposed method for the selected phenolic compounds

\begin{tabular}{|c|c|c|c|c|c|c|c|c|}
\hline \multirow{2}{*}{ Analyte } & \multirow{2}{*}{$\mathbf{L R}^{\mathbf{a}}$} & \multirow{2}{*}{$R^{2 b}$} & \multirow{2}{*}{$\operatorname{LOD}^{c}$} & \multirow{2}{*}{$\operatorname{LOQ}^{d}$} & \multirow{2}{*}{$E F \pm S D^{e}$} & \multirow{2}{*}{$E R \pm S D^{f}$} & \multicolumn{2}{|c|}{ RSD (\%)g } \\
\hline & & & & & & & Intra-day & Inter-day \\
\hline Phenol & $0.33-4000$ & 0.998 & 0.10 & 0.33 & $220 \pm 20$ & $44 \pm 4$ & 5 & 6 \\
\hline o-Cresol & $0.26-4000$ & 0.996 & 0.08 & 0.26 & $400 \pm 15$ & $80 \pm 3$ & 4 & 5 \\
\hline$m$-Cresol & $0.70-4000$ & 0.998 & 0.20 & 0.70 & $225 \pm 7$ & $45 \pm 3$ & 6 & 7 \\
\hline$p$-Cresol & $0.33-4000$ & 0.998 & 0.10 & 0.33 & $400 \pm 20$ & $80 \pm 4$ & 5 & 7 \\
\hline 4-Chlorophenol & $0.23-4000$ & 0.998 & 0.07 & 0.23 & $440 \pm 15$ & $88 \pm 3$ & 4 & 5 \\
\hline 2-Nitrophenol & $0.26-4000$ & 0.998 & 0.08 & 0.26 & $415 \pm 30$ & $83 \pm 6$ & 8 & 8 \\
\hline
\end{tabular}

a Linear range $\left(\mu g \mathrm{~L}^{-1}\right)$

${ }^{b}$ Coefficient of determination

cLimit of detection, $S / N=3\left(\mu g L^{-1}\right)$

${ }^{d}$ Limit of quantification, $S / N=10\left(\mu g L^{-1}\right)$

eMean enrichment factor \pm standard deviation, $(n=3)$

${ }^{\prime}$ Mean extraction recovery \pm standard deviation, $(n=3)$

${ }^{g}$ Relative standard deviation ( $\left.n=6, C=50 \mu \mathrm{g} \mathrm{L}^{-1}\right)$ for intra-day and $\left(n=4, C=50 \mu \mathrm{g} \mathrm{L^{-1 }}\right.$ ) for inter-day precisions

\subsubsection{Real sample analysis}

To demonstrate the performance of the proposed method, it was applied to the determination of the target analytes in four wastewater samples including input and output of treatment plant of Tabriz Petrochemical Company, output of the desalination unit and final output of refinery (both from Tabriz Refinery). After extracting the analytes with the proposed method and their determination by GC-FID, the analytes concentrations were calculated by standard addition method and shown in Table 2. The typical GC-FID chromatograms of blank, standard solution $\left(200 \mathrm{mg} \mathrm{L}^{-1}\right.$ of each derivatized analyte), output of the desalination unit refinery wastewater, input of the petrochemical wastewater, final output of refinery wastewater, and final output of petrochemical wastewater are shown in Figure 7. According the obtained results, none of the analytes were detected in final output of petrochemical wastewater and final output of refinery wastewater. While in the output of the desalination unit, some peaks are observed at retention times of the analytes that they can be related to phenol, $o$-cresol, $m$-cresol-, $p$-cresol, and 4-chlorophenol. repeatability for the developed technique is achievable. The calibration graph is linear in the broad concentration ranges for all selected analytes with coefficients of determination higher than 0.996. The LODs and LOQs calculated on the basis of signal to noise ratio $(\mathrm{S} / \mathrm{N})$ of 3 and 10, respectively, ranged from $0.07-0.20$ and $0.23-0.70 \mu \mathrm{g} \mathrm{L}^{-1}$, respectively. The EFs and ERs are between 220 and 440 and 44 and 88\%, respectively. Good repeatability, high EFs and ERs, and low LODs and LOQs are main advantages of the proposed method.
Also, in the input of petrochemical wastewater sample two peaks are observed at retention times of phenol and $\mathrm{m}$ cresol.

To confirm the obtained results, all samples were analyzed by GC-MS after performing the proposed method on the mentioned samples. The obtained typical total ions current (TIC) chromatogram for output of desalination unit of refinery along with the mass data are shown in Figure 8. The mass data confirmed the presence of the mentioned analytes in the samples. Matrix effect was studied through "added-found" method. For this purpose the samples were spiked at three different concentrations $(50,100$, and 500 $\mu \mathrm{g} \mathrm{L}^{-1}$ of each analyte) and analyzed by the proposed method. The obtained peak areas were compared with the corresponding peak areas in the chromatogram of deionized water added the same concentrations. The results of this comparison as relative recoveries are summarized in Table 3. As a result, matrix effect was only observed in input of petrochemical wastewater. To solve this problem, after testing different dilution ratios, input of the petrochemical wastewater was diluted at a ratio of 1:1 with deionized water to reduce its matrix effect.

Table 2. Analytes' contents of the samples determined by the proposed HLLE-DLLME-GC-FID method

\begin{tabular}{|c|c|c|c|c|}
\hline \multirow[b]{2}{*}{ Analyte } & \multicolumn{4}{|c|}{ Mean concentration of the analyte $\left(\mu \mathrm{g} \mathrm{L}^{-1}\right) \pm$ standard deviation $(n=3)$} \\
\hline & $\begin{array}{c}\text { Input of petrochemical } \\
\text { wastewater }\end{array}$ & $\begin{array}{c}\text { Final output of } \\
\text { petrochemical Wastewater }\end{array}$ & $\begin{array}{c}\text { Output of desalination } \\
\text { unit of refinery } \\
\text { wastewater }\end{array}$ & $\begin{array}{l}\text { Final output of } \\
\text { refinery wastewater }\end{array}$ \\
\hline Phenol & $2049 \pm 113$ & $\mathrm{ND}^{\mathrm{a}}$ & $788 \pm 43$ & ND \\
\hline o-Cresol & ND & ND & $243 \pm 11$ & ND \\
\hline$m$-Cresol & $31 \pm 2$ & ND & $109 \pm 6$ & ND \\
\hline$p$-Cresol & ND & ND & $91 \pm 5$ & ND \\
\hline 4-Chlorophenol & ND & ND & $74 \pm 3$ & ND \\
\hline 2-Nitrophenol & ND & ND & ND & ND \\
\hline
\end{tabular}

${ }^{a}$ Not detected 

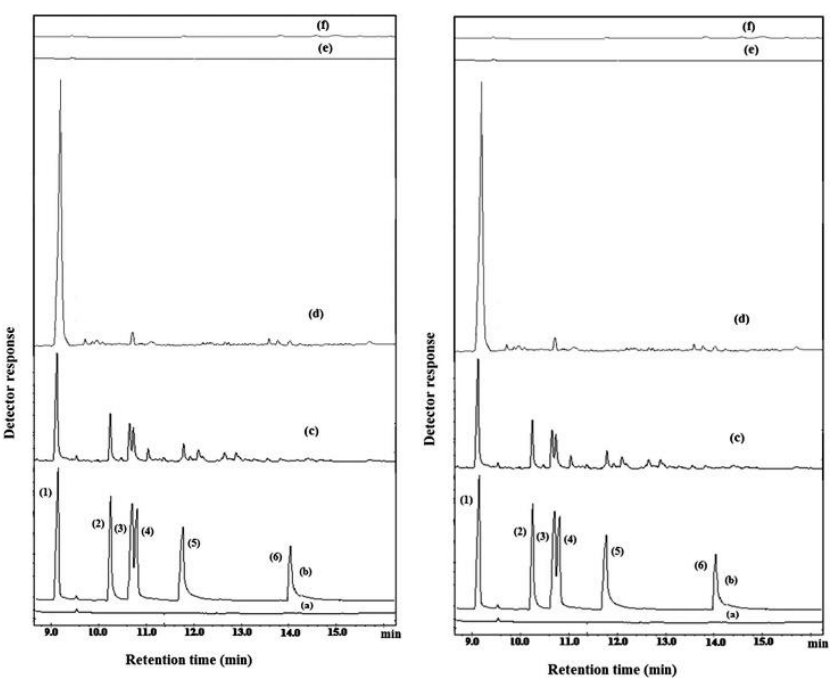

Figure 7. GC-FID chromatograms of: (a) blank, (b) standard solution of the derivatized phenolic compounds in 1,1,2-TCE (200 mg L-1, each phenolic compound), (c) output of desalination unit of refinery, (d) input of the petrochemical wastewater, (e)

final output of refinery wastewater, and (f) final output of petrochemical wastewater. All chromatograms, except (b) were obtained by applying the extraction method and injection $1 \mu \mathrm{L}$ of the sedimented organic phase into GC-FID. In chromatogram (b) direct injection $(1 \mu \mathrm{L})$ was used. Peaks identification: (1) phenol, (2) o-cresol, (3) $m$-cresol, (4) $p$-cresol, (5) 4-chlorophenol, and (6) 2-nitrophenol
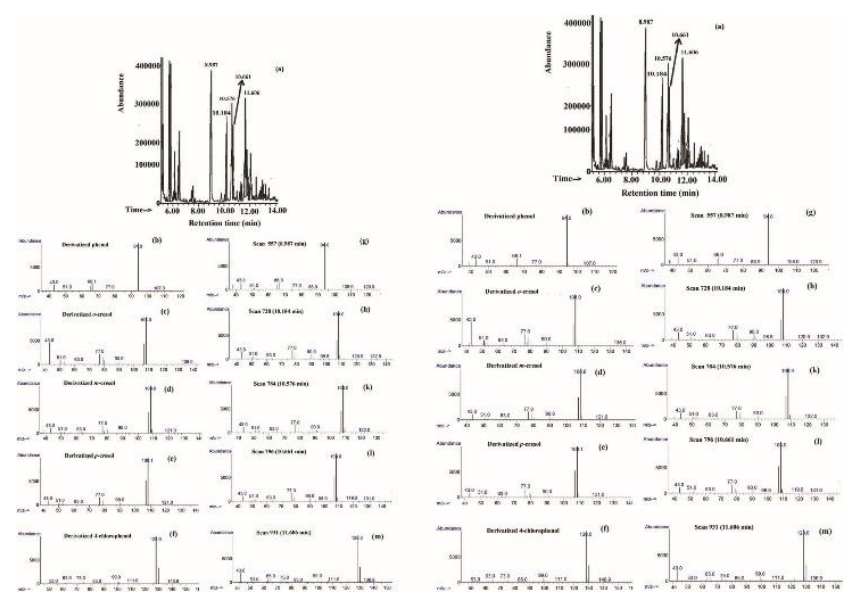

Figure 8. (a) GC-TIC-MS of output of desalination unit of refinery after performing the proposed method and mass spectra of derivatized (b) phenol, (c) $o$-cresol, (d) $m$-cresol, (e) $p$-cresol, and (f) 4-chlorophenol, and scans (g) 557 (retention time $8.987 \mathrm{~min}$ ),

(h) 728 (retention time $10.184 \mathrm{~min}$ ), (k) 784 (retention time $10.576 \mathrm{~min}$ ), (l) 796 (retention time $10.661 \mathrm{~min}$ ), and (m) 931 (retention time $11.606 \mathrm{~min}$ )

Table 3. Study of matrix effect in the studied samples. Analytes' contents of the samples were subtracted. All samples were used without dilution, except input of petrochemical wastewater which was diluted 1:1 with deionized water

\begin{tabular}{|c|c|c|c|c|}
\hline \multirow[b]{2}{*}{ Analyte } & \multicolumn{4}{|c|}{ Mean relative recovery $(\%) \pm$ standard deviation $(n=3)$} \\
\hline & $\begin{array}{c}\text { Input of petrochemical } \\
\text { wastewater }\end{array}$ & $\begin{array}{c}\text { Output of desalination } \\
\text { unit of refinery } \\
\text { wastewater }\end{array}$ & $\begin{array}{c}\text { Final output of } \\
\text { refinery } \\
\text { wastewater }\end{array}$ & $\begin{array}{c}\text { Final output of } \\
\text { petrochemical } \\
\text { wastewater }\end{array}$ \\
\hline \multicolumn{5}{|c|}{ All samples were spiked with each analyte at a concentration of $50 \mu \mathrm{g} \mathrm{L}^{-1}$} \\
\hline Phenol & $75 \pm 4$ & $89 \pm 5$ & $99 \pm 5$ & $88 \pm 5$ \\
\hline o-Cresol & $94 \pm 4$ & $71 \pm 3$ & $95 \pm 4$ & $94 \pm 4$ \\
\hline$m$-Cresol & $73 \pm 4$ & $90 \pm 6$ & $100 \pm 6$ & $89 \pm 6$ \\
\hline$p$-Cresol & $80 \pm 4$ & $83 \pm 4$ & $88 \pm 4$ & $93 \pm 5$ \\
\hline 4-Chlorophenol & $88 \pm 4$ & $89 \pm 4$ & $100 \pm 4$ & $72 \pm 3$ \\
\hline 2-Nitrophenol & $81 \pm 6$ & $99 \pm 8$ & $92 \pm 7$ & $95 \pm 8$ \\
\hline \multicolumn{5}{|c|}{ All samples were spiked with each analyte at a concentration of $100 \mu \mathrm{g} \mathrm{L}^{-1}$. } \\
\hline Phenol & $81 \pm 4$ & $97 \pm 5$ & $95 \pm 5$ & $90 \pm 5$ \\
\hline o-Cresol & $98 \pm 4$ & $78 \pm 3$ & $95 \pm 4$ & $92 \pm 4$ \\
\hline$m$-Cresol & $97 \pm 6$ & $87 \pm 6$ & $96 \pm 6$ & $96 \pm 6$ \\
\hline$p$-Cresol & $95 \pm 5$ & $97 \pm 5$ & $91 \pm 4$ & $93 \pm 5$ \\
\hline 4-Chlorophenol & $97 \pm 4$ & $97 \pm 4$ & $90 \pm 4$ & $92 \pm 4$ \\
\hline 2-Nitrophenol & $89 \pm 7$ & $92 \pm 7$ & $98 \pm 8$ & $86 \pm 7$ \\
\hline \multicolumn{5}{|c|}{ All samples were spiked with each analyte at a concentration of $500 \mu \mathrm{g} \mathrm{L}^{-1}$. } \\
\hline Phenol & $100 \pm 5$ & $81 \pm 4$ & $96 \pm 5$ & $97 \pm 5$ \\
\hline o-Cresol & $95 \pm 4$ & $87 \pm 4$ & $86 \pm 4$ & $100 \pm 4$ \\
\hline$m$-Cresol & $99 \pm 6$ & $98 \pm 6$ & $92 \pm 6$ & $99 \pm 6$ \\
\hline$p$-Cresol & $97 \pm 5$ & $98 \pm 5$ & $97 \pm 5$ & $99 \pm 5$ \\
\hline 4-Chlorophenol & $80 \pm 4$ & $90 \pm 4$ & $95 \pm 4$ & $92 \pm 4$ \\
\hline 2-Nitrophenol & $70 \pm 7$ & $88 \pm 7$ & $96 \pm 7$ & $94 \pm 8$ \\
\hline
\end{tabular}




\subsubsection{Comparison of the proposed method with other approaches}

For this purpose analytical characteristics of the proposed method including LOD, LR, RSD, and EF were compared with those of other relevant methods for determination of the phenolic compounds in aqueous samples. method exhibits low or comparable RSDs with others. The LODs of the proposed method are lower than those of other methods. In addition wide linear range was observed for calibration curve of all analytes. High EF is another advantage of the method compared to other approaches. These results are summarized in Table 4 . The current

Table 4. Comparison of the presented method with other methods used in determination of different phenolic compounds

\begin{tabular}{|c|c|c|c|c|c|c|c|}
\hline Analyte & Sample & $\mathrm{LR}^{\mathrm{a}}\left(\mu \mathrm{g} \mathrm{L}^{-1}\right)$ & $\operatorname{LOD}^{b}\left(\mu \mathrm{g} \mathrm{L}^{-1}\right)$ & $\mathrm{EF}^{\mathrm{c}}$ & RSD (\%) & Method & Ref. \\
\hline 2-Nitrophenol & $\begin{array}{l}\text { Water } \\
\text { samples }\end{array}$ & $50-300$ & 10 & 336 & 1.48 & $\begin{array}{l}\text { Hollow fiber-based } \\
\text { three-phase LPME-CE }\end{array}$ & (Sanaji et al., 2010) \\
\hline Phenol & Wastewater & $5-10000$ & 1.38 & - & 1.65 & LPME-GC-FID ${ }^{f}$ & $\begin{array}{c}\text { (Zhang and } \\
\text { Marzban, 2010) }\end{array}$ \\
\hline o-Cresol & & $5-10000$ & 1.97 & - & 3.58 & & \\
\hline$m$-Cresol & & 5-10000 & 1.34 & - & 0.96 & & \\
\hline Phenol & Wastewater & $5-200$ & 1.3 & 30 & 14.8 & DLLME-HPLC-DADg & (Saraji et al., 2010) \\
\hline 2-Nitrophenol & & $0.5-500$ & 0.4 & 97 & 16.6 & & \\
\hline $\begin{array}{c}\text { 4- } \\
\text { Chlorophenol }\end{array}$ & $\begin{array}{c}\text { Water } \\
\text { samples }\end{array}$ & $4-400$ & 2 & 383 & 4.7 & $\begin{array}{l}\text { DLLME-derivatization- } \\
\text { GC-ECD }\end{array}$ & $\begin{array}{c}\text { (Fattahi et al., } \\
\text { 2007) }\end{array}$ \\
\hline Phenol & $\begin{array}{l}\text { Aqueous } \\
\text { samples }\end{array}$ & $0.33-4000$ & 0.10 & 220 & 5 & $\begin{array}{l}\text { Derivatization-HLLE- } \\
\text { DLLME-GC-FID }\end{array}$ & This work \\
\hline$o$-Cresol & & $0.26-4000$ & 0.08 & 400 & 4 & & \\
\hline$m$-Cresol & & $0.70-4000$ & 0.20 & 225 & 6 & & \\
\hline$p$-Cresol & & $0.33-4000$ & 0.10 & 400 & 5 & & \\
\hline $\begin{array}{c}\text { 4- } \\
\text { Chlorophenol }\end{array}$ & & $0.23-4000$ & 0.07 & 440 & 4 & & \\
\hline 2-Nitrophenol & & $0.26-4000$ & 0.08 & 415 & 8 & & \\
\hline
\end{tabular}

aLinear range

bimit of detection

'Enrichment factor

${ }^{d}$ Relative standard deviation

eHollow fiber-based three phase liquid-phase microextraction-capillary electrophoresis

fLiquid-phase microextraction-gas chromatography-flame ionization detection

${ }^{g}$ Dispersive liquid-liquid microextraction-high performance liquid chromatography-diode array detector

${ }^{h}$ Dispersive liquid-liquid microextraction-derivatization-gas chromatography-electron capture detector

iDerivatization-homogeneous liquid-liquid extraction-dispersive liquid-liquid microextraction -gas chromatography-flame ionization detector

\section{Conclusions}

In this study, initially the studied phenolic compounds in aqueous samples were derivatized with acetic anhydride and then extracted and preconcentrated by coupling HLLE and DLLME methods. The derivatization process used in this study have some advantageous such as effective derivatization of the phenolic compounds and saving time. This method benefits the advantages of both HLLE and DLLME methods. Evaluation of the proposed method by its applying on real samples demonstrated that this method is a powerful analytical technique which provides high extraction efficiency, short extraction time, simplicity of operation, low cost, and low consumption of organic solvents. Accordingly, this method is appropriate for precise and accurate determination of the studied phenolic compounds in aqueous samples.

Abbreviations: DLLME, Dispersive liquid-liquid microextraction; EF, Enrichment factor; ER, Extraction recovery; FID, Flame ionization detector; GC, Gas chromatography; HLLE, Homogeneous liquid-liquid extraction; LOD, Limit of detection; LOQ, Limit of quantification; LPME, Liquid phase microextraction; MS, Mass spectrometry; RSD, Relative standard deviation.

\section{Acknowledgments}

This work was supported by the Research Council of Tabriz University.

\section{References}

Bagheri H., Babanezhad E. and Khalilian F. (2008), A novel sol-gelbased amino-functionalized fiber for headspace solid-phase microextraction of phenol and chlorophenols from environmental samples, Analytica Chimica Acta, 616, 49-55.

Bagheri H. and Saraji M. (2001), New polymeric sorbent for the solid-phase extraction of chlorophenols from water samples followed by gas chromatography-electron-capture detection, Journal of Chromatography A, 910, 87-93.

Ballesteros V., Gallego M. and Valcárcel M. (1990), Gas chromatographic determination of phenol compounds with automatic continuous extraction and derivatization, Journal of Chromatography A, 518, 59-67. 
Drinking Water Directive 80/778/EEC, Commission of the European Communities, Brussels, 1980.

Faraji H., Tehrani M.S. and Syed Wagif H. (2009), Preconcentration of phenolic compounds in water samples by novel liquid-liquid microextraction and determination by gas chromatography-mass spectrometry, Journal of Chromatography A, 1216, 8569-8574.

Farajzadeh M.A., Yadeghari A., Khoshmaram L. and Ghorbanpour H. (2014), Development and validation of a rapid and sensitive gas chromatographic method for the analysis of some phenolic compounds in vegetable oils, Analytical Methods, 6 , 5314-5321.

Fattahi N., Assadi Y., Milani Hosseini M.R. and Zeini Jahromi E. (2007), Determination of chlorophenols in water samples using simultaneous dispersive liquid-liquid microextraction and derivatization followed by gas chromatography-electron capture detection, Journal of Chromatography A, 1157, 23-29.

Fu S., Li F., Chu S.G. and Xu X.B. (2002), The determination of chlorophenols in waste water by capillary zone electrophoresis with an organic modifier, Chromatographia, $56,69-72$.

Gan T., Lv Zh., Sun J., Shi Zh. and Liu Y. (2016), Preparation of graphene oxide-wrapped carbon sphere@silver spheres for high performance chlorinated phenols sensor, Journal of Hazardous Materials, 302, 188-197.

Gan T., Wang Zh., Gao J., Sun J., Wu K., Wang H. and Liu Y.(2019). Morphology-dependent electrochemical activity of $\mathrm{Cu}_{2} \mathrm{O}$ polyhedrons and construction of sensor for simultaneous determination of phenolic compounds with graphene oxide as reinforcement, Sensors and Actuators, B, 282, 549-558.

Gan T., Wang Zh., Wang Y., Li X., Sun J. and Liu Y. (2017), Flexible graphene oxide-wrapped $\mathrm{SnO} 2$ hollow spheres with high electrochemical sensing performance in simultaneous determination of 4-aminophenol and 4-chlorophenol, Electrochimica Acta, 250, 1-9.

Ghorbanpour H., Yadeghari A., Khoshmaram L. and Farajzadeh M.A. (2014), Air-assisted liquid-liquid microextraction for simultaneous derivatization, extraction, and preconcentration of some phenolic compounds, Analytical Methods, 6, 7733-7743.

Halket J.M. and Zaikin V.G. (2004), Derivatization in mass spectrometry. 3. Alkylation (arylation), European journal of Mass Spectrometry, 10, 1-19.

Hu C., Chen B., He B. and Hu M. (2013), Amino modified multiwalled carbon nanotubes/polydimethylsiloxane coated stir bar sorptive extraction coupled to high performance liquid chromatography-ultraviolet detection for the determination of phenols in environmental samples, Journal of chromatography A, 1300, 165-172.

Kim S.H., Sun Y., Kaplan J.A., Grinstaff M.W. and Parquette J.R. (2015), Photo-crosslinking of a self-assembled coumarindipeptide hydrogel, New Journal of Chemistry, 39, 32253228.

Kujawski M.W., Bargańska Ż., Marciniak K., Miedzianowska E., Kujawski J.K., Ślebioda M. and Namieśnik J. (2014), Determining pesticide contamination in honey by LC-ESIMS/MS - Comparison of pesticide recoveries of two liquidliquid extraction based approaches, LWT-Food Science and Technology, 56, 517-523.
Llompart M., Lourido M., Landin P., Garcia-Jares C. and Cela R. (2002), Optimization of a derivatization-solid-phase microextraction method for the analysis of thirty phenolic pollutants in water samples, Journal of Chromatography A, 963, 137-148.

Nielson A.H., Allard A.S., Hynning P.A. and Remberger M. (1991), Distribution, fate and persistence of organochlorine compounds formed during production of bleached pulp, Toxicological and Environmental Chemistry, 30, 3-41.

Ou J., Hu L., Hu L., Li X. and Zou H. (2006), Determination of phenolic compounds in river water with on-line coupling bisphenol A imprinted monolithic precolumn with high performance liquid chromatography, Talanta, 69, 1001-1006.

Pierce A.E. (1968), Silylation of organic compounds, Rockford, Pierce Chemical Co.

Puig D. and Barcelo D. (1996), Determination of phenolic compounds in water and waste water, TrAC Trends in Analytical. Chemistry, 15, 362-375.

Rezaee M., Assadi Y., Milani Hosseini M.R., Aghaee E., Ahmadi F. and Berijani S. (2006), Determination of organic compounds in water using dispersive liquid-liquid microextraction, Journal of Chromatography A, 1116, 1-9.

Rodríguez I., MejutoV., Bollaín M.H. and Cela R. (1997), Evaluation of two solid-phase extraction procedures for the preconcentration of chlorophenols in drinking water, Journal of Chromatography A, 786, 285-292.

Rodríguez I., Turnes M.I., Mejuto M.C. and Cela R. (1996), Determination of chlorophenols at the sub-ppb level in tap water using derivatization, solid-phase extraction and gas chromatography with plasma atomic emission detection, Journal of Chromatography A, 721, 297-304.

Sanagi M.M, Miskam M., Ibrahim W.A.W., Hermawan D. and Aboul-Enein H.Y. (2010), Determination of partition coefficient and analysis of nitrophenols by three-phase liquid-phase microextraction coupled with capillary electrophoresis, Journal of Separation Science, 33, 21312139.

Sarafraz_Yazdi A., Rajab Dizavandi Z. and Amiri A. (2012), Determination of phenolic compounds in water and urine samples using solid-phase microextraction based on sol-gel technique prior to GC-FID. Analytical Methods, 4, 4316-4325.

Saraji M. and Bakhshi M. (2005), Determination of phenols in water samples by single drop microextraction folloed by Insyring derivatization and gas chromatography-mass spectrometricdetection, Journal of Chromatography A, 1098, 30-36.

Saraji M. and Marzban M. (2010), Determination of 11 priority pollutant phenols in wastewater using dispersive liquid-liquid microextraction followed by high-performance liquid chromatography-diode-array detection, Analytical and Bioanalytical Chemistry, 396, 2685-2693.

Shang H.B., Yang C.X. and Yan X.P. (2014), Metal-organic framework UiO-66 coated stainless steel fiber for solid-phase microextraction of phenols in water samples, Journal of Chromatography A, 1357, 165-171.

Sojo L.E. and Djauhari J. (1999), Determination of chlorophenolics in waters by membrane solid-phase extraction: Comparison between $\mathrm{C} 18$ and activated carbon membranes and between modes of extraction and elution, Journal of Chromatography A, 840, 21-30. 
Turnes I., Rodriguez I., Garcia C.M. and Cela R. (1996), Determination of chlorophenols in drinking water with high resolution gas chromatography-tandem mass spectrometry, Journal of Chromatography A, 743, 283-292.

Villar-Navarro M., Ramos-Payán M., Pérez-Bernal J.L., FernándezTorres R., Callejón-Mochón M. and Bello-López M.Á. (2012), Application of three phase hollow fiber based liquid phase microextraction (HF-LPME) for the simultaneous HPLC determination of phenol substituting compounds (alkyl-, chloro- and nitrophenols), Talanta, 99, 55-61.

Visscher A.D., Eenoo P.V., Drijvers D. and Langenhove H.V. (1996), Kinetic Model for the Sonochemical Degradation of Monocyclic Aromatic Compounds in Aqueous Solution, The Journal of Physical and. Chemistry, 100, 11636-11642.

Zhang T., Chen X., Liang P. and Liu C. (2006), Determination of phenolic compounds in wastewater by liquid-phase microextraction coupled with gas chromatography, Journal of Chromatographic Science, 44, 619-624.

Zhao R.S., Wang X., Yuan J.P. and Zhang L.L. (2009), Solid-phase extraction of bisphenol $A$, nonylphenol and 4-octylphenol from environmental water samples using microporous bamboo charcoal, and their determination by HPLC. Microchimica Acta, 165, 443-447.

Zhou F., Li X. and Zeng Z. (2005), Determination of phenolic compounds in wastewater samples using a novel fiber by solid-phase microextraction coupled to gas chromatography, Analytica Chimica Acta, 538, 63-70. 